\title{
Robust Facial Alignment with Internal Denoising Auto-Encoder
}

\author{
Decky Aspandi, Oriol Martinez, Federico Sukno and Xavier Binefa \\ Department of Information and Communication Technologies \\ Pompeu Fabra University \\ Barcelona, Spain \\ \{decky.aspandilatif, oriol.martinez, federico.sukno, xavier.binefa\}@upf.edu
}

\begin{abstract}
The development of facial alignment models is growing rapidly thanks to the availability of large facial landmarked datasets and powerful deep learning models. However, important challenges still remain for facial alignment models to work on images under extreme conditions, such as severe occlusions or large variations in pose and illumination. Current attempts to overcome this limitation have mainly focused on building robust feature extractors with the assumption that the model will be able to discard the noise and select only the meaningful features. However, such an assumption ignores the importance of understanding the noise that characterizes unconstrained images, which has been shown to benefit computer vision models if used appropriately on the learning strategy. Thus, in this paper we investigate the introduction of specialized modules for noise detection and removal, in combination with our state-of-the-art facial alignment module and show that this leads to improved robustness both to synthesized noise and in-the-wild conditions. The proposed model is built by combining two major subnetworks: internal image denoiser (based on the Auto-Encoder architecture) and facial landmark localiser (based on the inception-resnet architecture). Our results on the 300-W and Menpo datasets show that our model can effectively handle different types of synthetic noise, which also leads to enhanced robustness in real-world unconstrained settings, reaching top state-of-the-art accuracy.
\end{abstract} tion.

Keywords-Facial Alignment, Auto Encoder, Noise Normaliza-

\section{INTRODUCTION}

Facial alignment aims to detect a set of facial landmark positions which can later be used for several facial analysis applications [1]. The development of facial alignment models is growing rapidly with the availability of large facial landmarked dataset such as 300-W [2] and Menpo [3] dataset. This has made it possible the development of powerful deep learning models that have pushed forward the alignment accuracy and are considered the current state of the art [4], [5].

However the performance of current facial alignment models can severely deteriorate when dealing with images in highly unconstrained conditions, e.g. extreme pose or illumination changes, large occlusions [6] or, in general, whenever the test images can be considered to show less favorable conditions than those available for training. In other words, we may say that under challenging conditions, test images contain some form of distortion or noise that will impair the performance of facial alignment models. This limits the real-life applicability of such models to in-the-wild images which naturally contain such challenging conditions [7], [8]. While there have been attempts to improve the robustness of facial alignment models to target in-the-wild data [9], [6], most of them have done so without modeling the effects of noise in their formulation. Nevertheless, understanding and incorporating such effects within the model training has proven beneficial to improve performance in other facial analysis tasks [10], [7], [11].

To address the above shortcomings, in this paper we investigate the introduction of specialized modules for noise detection and removal, in combination with our state-ofthe-art facial alignment module and show that this leads to improved robustness both to synthesized noise and in-thewild conditions. Our model is built by combining two major subnetworks: internal images denoiser and facial landmark localiser which work in parallel and can be trained endto-end. We adopt the Auto-Encoder architecture [12] with skip connection for the internal denoising, and the inceptionresnet architecture [13] for the facial landmark. We train our models with four of the most general image noise models: resolution down-sampling, gaussian blur, gaussian noise, and pixel intensities scaling. We show that the proposed architecture can successfully achieve robustness to these types of noise but also, and more importantly, we also find that such noise models are sufficient to train a network that achieves top state-of-the-art performance under real acquisition conditions, by testing on the 300-VW dataset, Specifically, our contributions in this paper are:

1) To the best of our knowledge, we are the first to investigate the robustness of current state of the art facial alignment methods upon the introduction of synthetic image noise.

2) We propose a novel network for robust facial alignment that is capable to produce accurate facial landmark estimates on unconstrained images by means of its internal denoising strategy.

3) We show that our model reach the state of the art results on the most challenging category of $300-\mathrm{VW}$ videos datasets, both on the single image alignment and facial landmark tracking. 


\section{RELATED WORK}

Face alignment has attracted considerable attention due to its importance for several applications, such as face recognition[14], head pose estimation[15], facial reenactment [16] and others. This task is generally conducted by estimating a predefined set of landmark positions which provide a structure representation of the facial geometry. Traditionally, facial landmarks have been estimated using either the shape and appearance models [17], [18] or regression based models [19], with the latter offering some advantage due to its computational efficiency [20]. Recent examples of regression-based models include the work of Kazemi and Sullivan [21], who use a cascade of regression functions to efficiently regress the landmark locations, and Zhu et al [22] who refined the regressors cascade with coarse to fine search.

The recent availability of large datasets such as 300-w [2] and Menpo [3] allows for large scale data modeling and development of deep learning-based models that benefit from these load of data. For instance, the work of Bulat and Tzimiropoulos [4] used multiple hourglass shaped convolutional networks with heatmap-guided layers to predict the final facial landmarks, while Zhang et al. [23] enforced the facial attributes as auxiliary features to their cascaded convolutional network to improve landmark estimates. These deep learning models currently hold the state of the art accuracy both on the single image facial alignment [2] and on the facial landmark tracking tasks [1].

Despite the maturity of current facial alignment models, there are still challenges for them to work on images with large appearance variations due to heavy occlusion, severe pose or illuminations conditions, etc [6]; especially on real world application targeting highly unconstrained settings [7]. Thus, there has been growing interest to improve the performance of facial models under such settings, including efforts such as iterative initialization of regression cascades to minimize the impact of outliers [9] or the combination of data- and model-driven estimators to enhance the robustness of landmark detection [6]. However, most efforts have focused on building robust feature extractors with the assumption that the model will be able to discard the noise and select only the meaningful features.

In contrast, we approach the problem differently by focusing on modelling image noise by means of our internal denoiser network and further minimizing it alongside of building an accurate facial landmark feature extractor. This strategy can be justified by recent findings such as those from Dong et al. [10], who aggregated different styles of images using Generative Adversarial Networks to improve their landmark estimates, revealing that even the slight color style variations between the same images can impact the accuracy of landmark estimations. Similar findings have been reported in other facial analysis tasks, such as on the work from Zhou et al. [8], who evaluated several facial detection models with synthetic noise, and Goswami et al. [11] who investigated the robustness of facial classification to synthetically distorted images. The findings in these reports have led us to hypothesize that modeling certain types of noise may help to characterize the behavior of facial analysis systems with unconstrained images and incorporating such noise modeling into the training process could improve the robustness of their results.

\section{FACE Alignment with De-Noiser Network (FADENN)}

We argue that given a noisy input image, the final landmark estimation can be improved by first minimizing the existing noise and only then passing the image to any facial landmark estimator. To achieve this, we adopt a modular approach involving two major subnetworks to build our Face Alignment with De-Noiser Network (FADeNN) network: Internal Image Denoiser (IID) and Facial Landmark Localiser $(F L L)$ networks. Given the input image $I$, which can be either a clean or contain some unknown amount of noise, the IID network will be trained to internally detect and model the existing and, if required, clean the input image to generate a normalized image that will be fed to the FLL network to estimate $n$ landmark coordinates $\mathbf{l}_{I}$ $\left\{\hat{x}_{1} \ldots \hat{x}_{n}, \hat{y}_{1} \ldots \hat{y}_{n}\right\} \in \mathbb{R}_{>0}$.

$$
l_{I}=F A D e N N(I)=F L L(I I D(I))
$$

\section{A. Internal Image Denoiser (IID)}

On this work, we explore two different models of IID : Direct Denoising AutoEncoder (Figure 1) and ClassifierSpecialized Denoiser (Figure 2). In both cases, the denoiser core is based on the Hourglass shaped Auto-Encoder Architecture (DAE) with skip connection [24]. The structure of our $D A E$ is similar on the work of [12] with a few additional mirror layers on both encoder and decoder parts. The code of the modified $D A E$ is available at https://github.com/deckyal/FADeNN.

Direct Denoising AutoEncoder: we first explore the most straightforward approach, which consists of directly attempting to denoise any new input image with the same model. The basic operation of direct denoising can formally be expressed as follows :

$$
I I D(I)=D A E_{\Phi^{1}}(I)=e n c_{\Phi^{1}}\left(\operatorname{dec}_{\Phi^{1}}(I)\right)
$$

where $\phi^{1}$ are the parameters learned for subnetwork $D A E$ consisting of coupled encoder enc(x) and decoder $\operatorname{dec}(x)$ layers. Due to the encoding and decoding operations, the original image will not be perfectly recovered after being processed by this network. In the ideal case, the output will be just a noise-free version of the input. However, in the cases when the input image is clean (i.e. without noise), the model may add itself a small amount of artificial noise due 


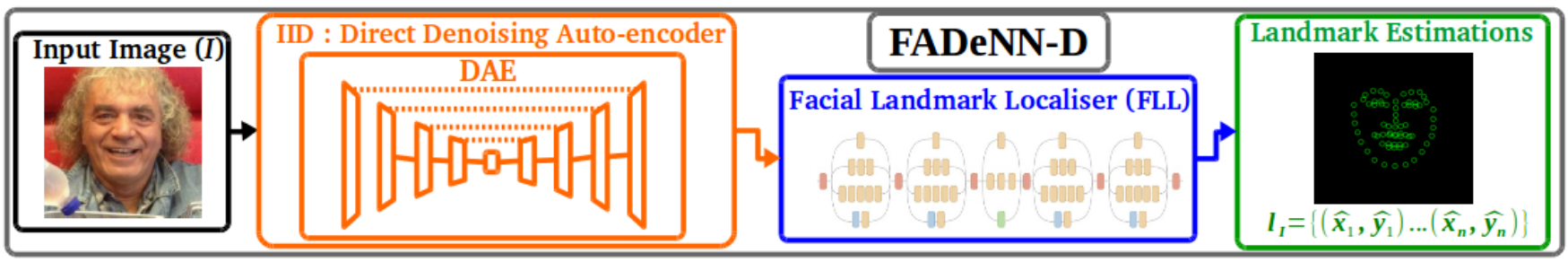

Figure 1. General overview of our robust facial alignment consisting two major subnetworks: Internal Image Denoiser and Facial Landmark Localiser.

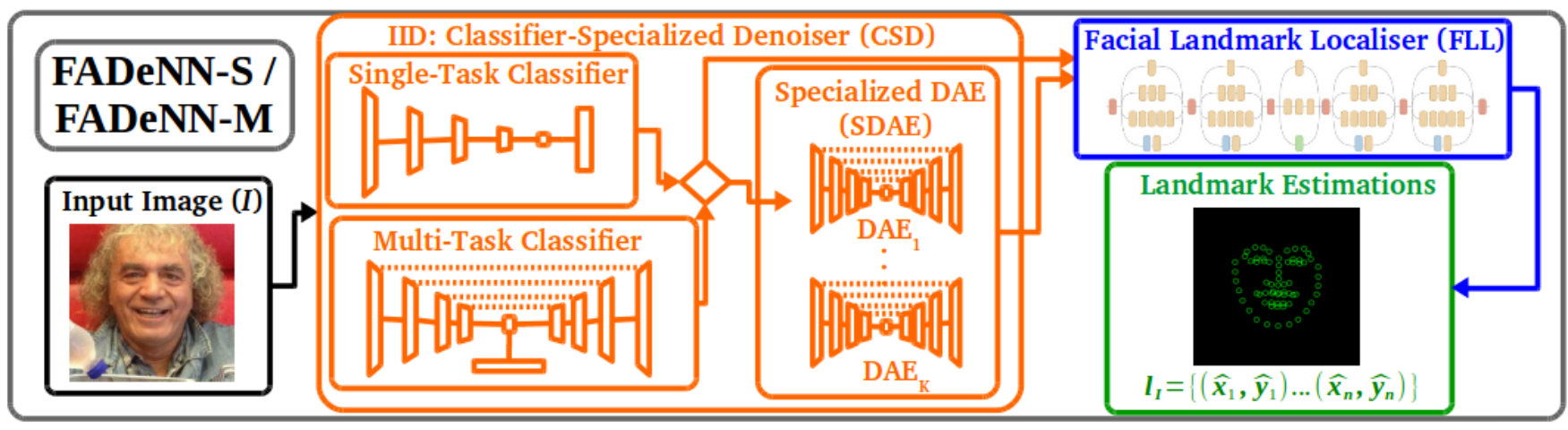

Figure 2. The structure of FADeNN-S and FADeNN-M which incorporates more deliberate Classifier-Specialized DAE.

to this imperfect reconstruction. To tackle this issue, below we propose our second denoising model.

Classifier - Specialized Denoiser (CSD) is built with the introduction of a Noise Classifier (NC) network and Multiple Specialized DAE (SDAE) subnetworks which work in tandem to remedy the limitation of direct DAE. The main concept of CSD is to perform the denoising selectively based on the detected condition of the input image to trigger separate specialized denoiser sub-models. The formulation of this IID block is as follows:

$$
I I D(I)=S D A E_{\phi^{3}}\left(N C_{\phi^{2}}(I), I\right)
$$

Given the number of $K$ known noise class, the $N C$ will estimate the probability that noise class $c$ is present in the input image $I$ :

$$
\begin{gathered}
N C_{\phi^{2}}(I)=\operatorname{argmax}\left\{s_{1}, s_{2} . . s_{K}\right\} \\
s_{k}=\left(\frac{e^{W_{\phi_{k}^{2}}^{N C} \odot \operatorname{conv} v_{\phi_{k}^{2}}^{N C}(I)}}{\sum_{k=1}^{K} e^{W_{\phi_{k}^{N C}}^{N C} \odot \operatorname{conv} v_{\phi_{k}^{2}}^{N C}(I)}}\right)
\end{gathered}
$$

Where $W^{N C}$ are the multinomial bottleneck regression layers parameterized by $\Phi^{2}, s_{k}$ is the score for specific noiseclass $k$ estimated for the current input $I$, and conv is the set of convolutional layers for $N C$. If the classifier detects noise in the input image, then $c>0$ and the image is denoised by one of the specialized denoisers $\left\{D A E_{1}, D A E_{2} \ldots D A E_{K}\right\}$; each of these blocks can be trained to capture a different type of noise.

$$
S D A E_{\phi^{3}}(c, I)=\left\{\begin{array}{l}
D A E_{\phi_{c}^{3}}(I), \text { if } c>0 \\
I, \text { otherwise }
\end{array}\right.
$$

Where $\Phi_{c}^{3}$ is the parameter learned for the specific DAE of class $c$. Otherwise, $c=0$ and the denoising process is skipped to avoid unnecessarily distorting the input image. The idea behind our CSD network follows a similar spirit to the work in [25], however we used distinct specialized subnetworks instead of specialized internal layers.

As observed in Fig. 2, we explored two architecture variants for our noise classifier: Single-Task Classifier $(S T C)$ and Multi-Task Classifier $(M T C)$. The differences between $S T C$ and $M T C$ are that $M T C$ uses additional reconstruction loss as regularizer [26], [4] alongside the classification loss, and $M T C$ uses full encoder-decoder architecture similarly to $D A E$ while $S T C$ uses only the encoder part.

\section{B. Facial Landmark Localiser (FLL)}

We build our $F L L$ from our recently published Composite Recurrent Convolution Tracker $C R C T$ [27]. Specifically, we adopt the landmark localization subnetwork from $C R C T$, based on the Inception-Resnet [13] architecture, which we modified by replacing the last layer with new bottleneck regression layers. ${ }^{1}$. The landmark localization procedure can

\footnotetext{
${ }^{1}$ Our modified inception resnet network is publicly available on: https://github.com/deckyal/RT
} 
be expressed mathematically as below:

$$
\mathbf{l}_{I}=F L L_{\Phi^{4}}(X)=W_{\Phi^{4}}^{I N C} \odot \operatorname{res}_{\Phi^{4}}(X)
$$

where FLL consists of the Inception-Resnet (res) and a regression layer of weight matrix $W^{I N C}$ parameterized by $\Phi^{4}$. The input to $F L L$ is the clean image $X$, which would be obtained as $X=I I D(I)$ in the general case.

\section{Overall Models and loss functions}

To provide a comprehensive analysis of the denoising alternatives explored in this paper, we provide results for three different models. We refer to these models based on their $I D D$ subnetworks :

1) FADeNN-D which uses direct $D A E$.

2) FADeNN-S which uses the combination of $S T C$ and $S D A E$.

3) FADeNN-M which uses $M T C$ and $S D A E$.

We use standard $\ell^{2}$ loss to train $F L L$ while the loss functions for each of the $I I D$ options are as follows :

$$
\begin{aligned}
& F A D e N N-D: \\
& \mathbf{L}_{\mathbf{I I D}}=\min \ell^{2}(X, D A E(\hat{X})), \\
& F A D e N N-S: \\
& \mathbf{L}_{\mathbf{I I D}}=\min (H(c, S T C(I))), L_{S D A E}, \\
& F A D e N N-M: \\
& \mathbf{L}_{\mathbf{I I D}}=\min \lambda_{1}(H(c, M T C(I))+ \\
&\left.\lambda_{2} \ell^{2}(I, M T C(I))\right), L_{S D A} .
\end{aligned}
$$

where $\hat{X}$ is a noisy version of $X$ (added synthetically), $H(x)$ is the cross entropy loss and $\lambda$ is the regularizer parameter for each term. We use $I$ to indicate both clean and noisy inputs $\left(X\right.$ and $\hat{X}$ ) and $L_{S D A}$ is the loss of the specialized $S D A$ networks:

$$
\left.\mathbf{L}_{\mathbf{S D A}}=\min _{\forall D \in S D A E} \ell^{2}(X, D(\hat{X}))\right)
$$

\section{Training setup}

We train our model progressively by first training each internal $I D D$ and $F L L$ component individually, and then jointly training them for further fine-tuning. We utilized the $300-\mathrm{W}$ [2] training dataset for both $I D D$ and FLL. We also introduce data augmentation procedures of $-45^{\circ}$ to $45^{\circ}$ degree rotations and horizontal flipping, with additional artificial stripping to simulate occlusions for training $F L L$. Finally, we set our $\lambda$ value to 0.5 , and train our model using ADAM optimizer [28] with initial learning rate of 0.0001 and progressive decaying every 1000 iterations.

\section{EXPERIMENTS}

\section{A. Image Degradation Models}

To systematically evaluate the impact of image degradation on the final landmark estimations, we generate synthetically distorted images and evaluate their effect on the final landmark estimation of each model. We generate these distorted images by perturbing the original image with specific types of noises. We consider four types of noise: three of them following the recent work by Zhou et al. [8] plus an additional noise class consisting of downsampling. Specifically, each noise class was obtained as follows:

1) Down-sampling. We down-sampled images successively by a factor of 2.0 , resulting in images with half, one-quarter and one-eight of the original image resolution.

2) Gaussian-Blurring. We added Gaussian Blur by convolving the input image with two dimensional Gaussian filters with $\sigma_{g b} \in\{1,3,5\}$.

3) Gaussian-Noise. We added random pixel colors distributed according to normal distributions with zero-mean and standard deviation $\sigma_{g n}^{2} \in$ $\{0.001,0.005,0.01\}$.

4) Color Scaling. We scaled the original pixel intensities linearly by a factor $s \in\{0.8,0.5,0.2\}$ from the original intensities.

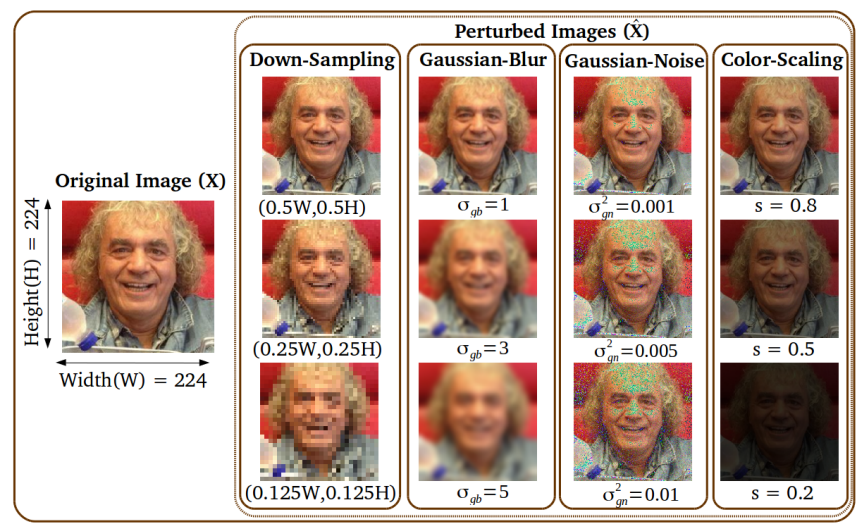

Figure 3. Example of a training image and its noise-distorted versions with specific types of noise.

Examples of the original and distorted images for each type of noise are depicted in Figure 3. Once trained, the robust models would be expected to produce small errors in the landmark estimates when given either normal or distorted input images.

\section{B. Datasets and Experimental settings}

To facilitate fair comparison, we used the standard benchmark of 300-W [2] and Menpo 2D test datasets [3] to evaluate the robustness of the evaluated models. We cropped and added synthetic noise to the original images on both datasets following section IV-A and named them as $300 \mathrm{~W}-T e s t-N x$ and Menpo-Test-Nx respectively, with $x$ standing for the type of noise that was added. We followed the standard testing procedure as in [3], [29] consisting of initializing all models with the bounding box obtained from the groundtruth points and quantitatively evaluating their final landmark 

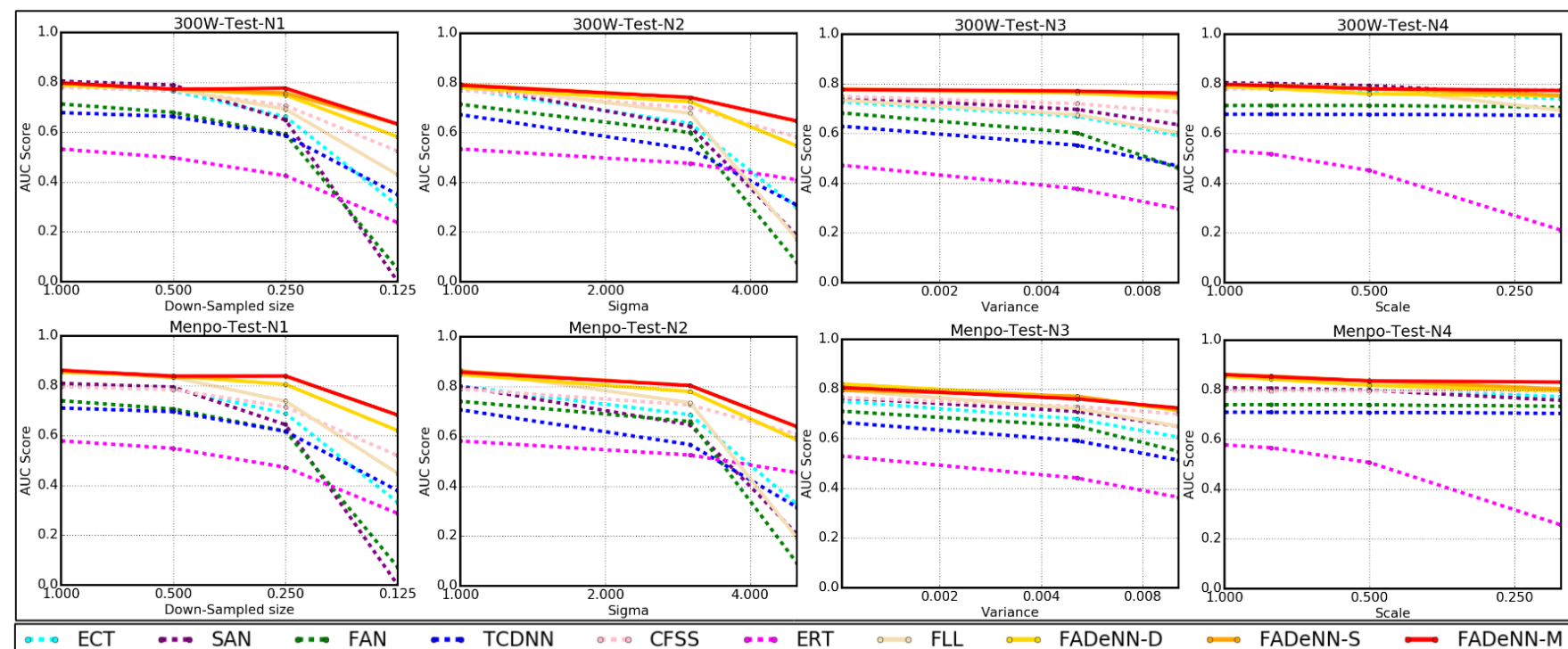

Figure 4. The AUC value degradation of the evaluated models on perturbed images with different noise levels.

estimations with Area Under the Curve (AUC) and Failure Rate (FR) for Normalized Mean Error (NME) by Facial Bounding Box scores up to 0.08 .

To further evaluate the robustness of our models in realworld conditions, we also tested our model trained with the noisy datasets on the Category 3 from $300-\mathrm{VW}$ dataset (the most challenging one). This subset contains low quality images with several illuminations, blurry images and challenging poses which are ideal to evaluate the robustness of the models [5], [1]. We refer to this dataset as 300-VW-3 and produce also a modified version with cropped images according to the facial bounding boxes 300-VW-3-C.

\section{Impact of Synthetically Degraded Images on Landmark Estimations}

To evaluate the robustness to degraded images of our model we report our results under 3 different configurations: $F A D e N N-D, F A D e N N-S$ and $F A D e N N-M$, as detailed in Section III-C. Additionally, we also introduce stand-alone $F L L$ to establish a baseline for the performance of our model with no denoising block at all. We compare our results with respect to six other facial alignment models. The first four consists of state of the art deep-learning based models: Robust Estimation-Correction-Tuning (ECT) Network [6], Style Aggregated Neural Network (SAN) [10], Facial Alignment Network (FAN) model [4] and Task Constrained Convolution Deconvolution Network (TCDCN) [23]. While the other two are traditional hand-crafted based models of facial alignment: Ensemble Regression Trees (ERT)[21] and Coarse to Fine Shape Searching (CFSS)[22].

On figure 4 we show the impact of each type of noise in the performance of the different models (full AUC and FR values of each models on each graph can be seen in the supplementary material). The deep learning models, including our baseline $F L L$ model are quite sensitive to both image down-sampling and Gaussian blurring. This can be seen on the rapid AUC score drop especially at the highest levels of distortion (one eighth of image resolution and gaussian blur with $\sigma_{g b}=5$ ). The [6] method on the other hand performs slightly better compared with other models given that it is designed to be robust to noise, albeit its performance is still in par with our base model FLL. We also see that the hand-crafted methods do not experience so significant performance drop and, indeed, CFSS [22] shows quite competitive results. For the gaussian and color scale noise, most models showed only moderate degradation with the exception of ERT, which performed slightly worse than the rest. This color and gaussian noise tolerance may be attributed to the internal image normalization steps used in the pre-processing pipelines of most methods. These results are consistent with findings from [8], suggesting universal weakness of current models against blur and resolution changes which may be due to lacks of blurry features in the designed or learned filter banks.

Further analysis of our own models performance, we find that our base FLL model performs comparatively well, on par with other state-of-the art models in spite of its simplicity, probably as a result of our rigorous transfer learning setup. In the heavily blurred and down-sampled images however, it still fails to locate landmark accurately (Figure 5 which is avoided by our $F A D e N N$ models thanks to their internal denoiser blocks (Figure 5). However, on the reasonably clean image (first row of Figure 5.b), we observe a slight accuracy drop from $F L L$ to $F A D e N N$ - D, which is explained by the use of the direct approach in which the $I I D$ 


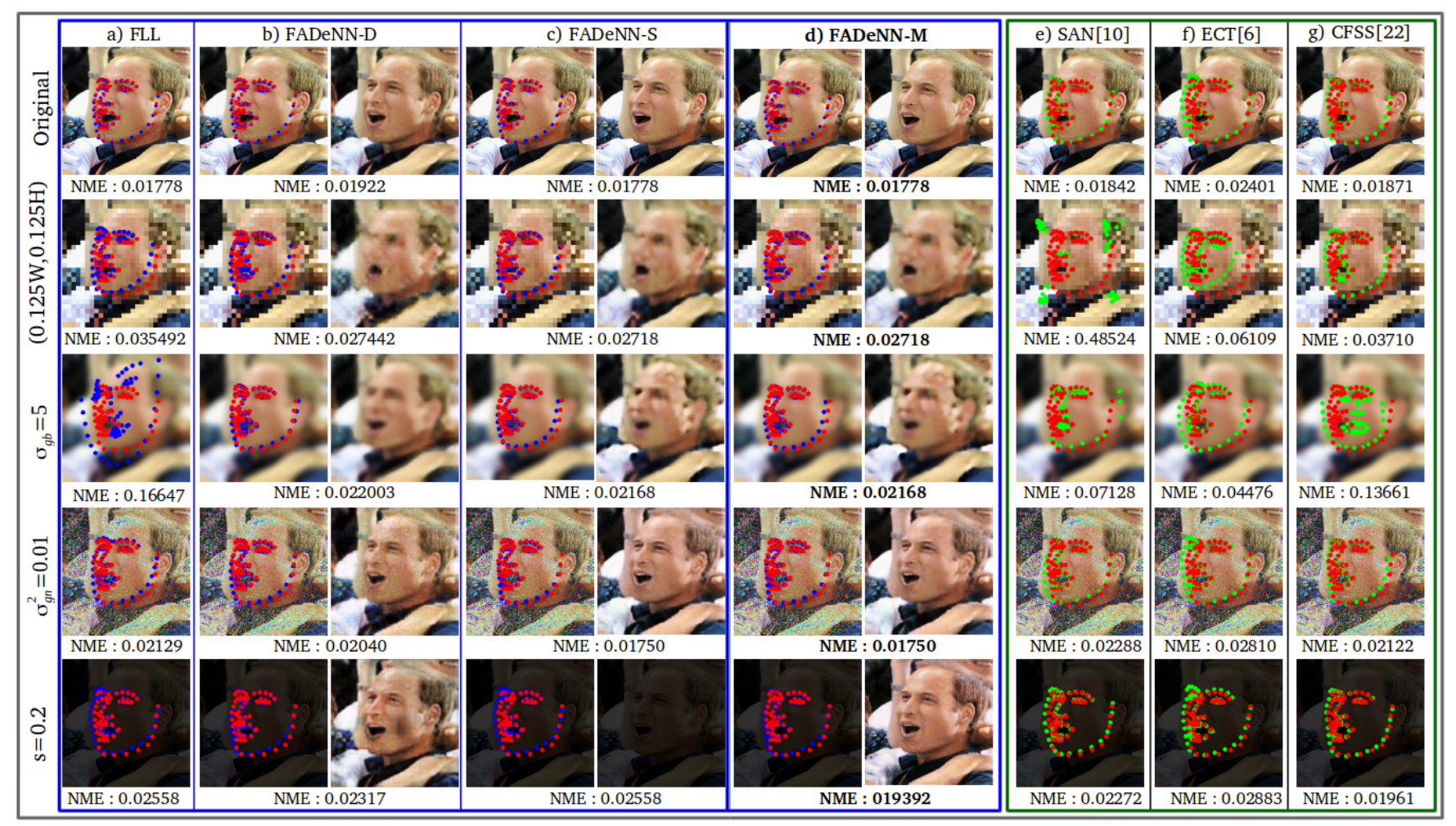

Figure 5. Example of facial landmark estimations. a) FLL, b) FADeNN-D, c) FADeNN-S, d) FADeNN-M, e) SAN[10], f) ECT[6], g) CFSS[22]

block attempts to denoise all input images, even when they may not require it. This is further solved by the selective denoising approach of FADeNN-S and FADeNN-M with even cleaner denoised image. Finally we find that $F A D e N N-M$ works slightly better than $F A D e N N-S$ because its classification scheme is more accurate in correctly identifying the type of denoising required for each input image.

\section{Comparison on the 300-VW Test dataset, category 3}

To compare the performance of our model under realworld conditions, in which the noise of the input images cannot be explicitly synthesized for training, we performed further experiments using the $300-\mathrm{VW}$ dataset. We considered two different settings: facial landmark localization on single images (using the cropped dataset 300-VW-3-C) and facial landmark tracking (on the original 300-VW-3 dataset, without cropping). For the latter, we convert our model to perform model-free tracking [29] by using the result from the previous frame as initialization for the current one. In case of fitting failure, the external facial detector [30] will be called for re-initialization. Fitting failure is automatically determined by means of an independent facial logistic regressor over the patches around landmark estimates as in [29]. Table I summarizes the results obtained by our method as well as by state-of-the-art alternatives (corresponding
AUC curves can be seen in the supplementary material). For the single-image setting we compare our results to the same methods used for comparison in the previous section, while for the tracking setting we compare our models with to the competitors of the original challenge in [1] and also to MD_CFSS and ME_CFSS, which were the highestperforming methods in the recent evaluation from [29].

We can see that our model achieves state-of-the-art accuracy, with a slight improvement with respect to the bestperforming alternatives, and at the same time produces far lower failure rates than all other compared methods. This behavior is consistent across both the single-image and the tracking settings. As in the experiments reported under synthetic noise in the previous section, we find that our base model FLL produces a comparable accuracy to other stateof-the-art models. Furthermore, with the introduction of the IID block, the result is progressively improved. The best performance is achieved by the FADeNN-M model.

We can also validate our result by visual inspection on Figure 6, where our models consistently provide the most accurate facial landmark estimates under very challenging conditions: strong illumination differences on the first row, blurry images on the second row, and also partially occluded facial parts on the third row. We can also see that the use of the internally normalized image on $F A D e N N-M$, even though not perfect, helps to correct the position of several 
landmark points, explaining its effectiveness.

\begin{tabular}{l|c|c|c|c}
\hline \multirow{2}{*}{ Method } & \multicolumn{2}{|c}{ Single-Image } & \multicolumn{2}{c}{ Tracking } \\
\cline { 2 - 5 } & AUC & FR & AUC & FR \\
\hline \hline ECT [6] & 0.763 & 0.037 & - & - \\
SAN [10] & 0.747 & 0.038 & - & - \\
FAN [4] & 0.733 & 0.015 & - & - \\
TCDNN [23] & 0.728 & 0.034 & - & - \\
CFSS [22] & 0.754 & 0.016 & - & - \\
ERT [21] & 0.680 & 0.120 & - & - \\
\hline MD_CFSS [29] & - & - & 0.726 & 0.075 \\
ME_CFSS [29] & - & - & 0.659 & 0.114 \\
Yang [31] & - & - & 0.710 & 0.045 \\
Jinwei [5] & - & - & 0.617 & 0.048 \\
Uricar [32] & - & - & 0.574 & 0.080 \\
Xiao[33] & - & - & 0.695 & 0.074 \\
Raja [34] & - & - & 0.659 & 0.083 \\
Wu[35] & - & - & 0.602 & 0.131 \\
\hline FLL & 0.752 & 0.017 & 0.711 & 0.022 \\
FADeNN-D & 0.756 & 0.015 & 0.718 & 0.020 \\
FADeNN-S & 0.760 & 0.013 & 0.726 & 0.020 \\
FADeNN-M & $\mathbf{0 . 7 6 4}$ & $\mathbf{0 . 0 1 2}$ & $\mathbf{0 . 7 2 9}$ & $\mathbf{0 . 0 1 9}$ \\
\hline
\end{tabular}

Table I

AUC AND FR SCORE ON THE 300-VW DATASET, CATEGORY 3RD

\section{CONCLusions}

In this paper, we evaluate the impact of synthetically degraded images on the performance of current facial alignment models and further use this understanding to build a robust facial alignment modes. We do this by building a deep neural network composed of a state-of-the-art Facial Landmark Localisation network in combination with an internal image denoising network.

Our systematic experiments with synthetically added noise reveal that the addition of synthetic noise degrades the performance of all the compared facial alignment models. In particular, the accuracy of deep learning models is severely degraded in the case of blurring and down-sampling but seems more resistant to gaussian noise and color scaling. In contrast, our proposed $F A D e N N$ models show enhanced robustness to all types of tested noise while achieving toplevel accuracy. Remarkably, we also find that models trained with simple types of synthesized noise maintain their robustness to challenging input images from real-World datasets, such as 300-VW (category 3). In such in-the-wild settings, our model internally estimates a cleaned-up version of the input image before performing landmark localisation, which leads to significantly lower failure rates than competing approaches while maintaining top state-of-the-art accuracy.

\section{ACKNOWLEDGMENTS}

This work is partly supported by the Spanish Ministry of Economy and Competitiveness under project grant TIN2017-90124-P, the Ramon y Cajal programme, the Maria de Maeztu Units of Excellence Programme (MDM-20150502) and the donation bahi2018-19 to the CMTech at the UPF.

\section{REFERENCES}

[1] J. Shen, S. Zafeiriou, G. G. Chrysos, J. Kossaifi, G. Tzimiropoulos, and M. Pantic, "The first facial landmark tracking in-the-wild challenge: Benchmark and results," in 2015 IEEE ICCVW, pp. 1003-1011.

[2] C. Sagonas, G. Tzimiropoulos, S. Zafeiriou, and M. Pantic, "300 faces in-the-wild challenge: The first facial landmark localization challenge," in 2013 IEEE ICCVW, pp. 397-403.

[3] S. Zafeiriou, G. Trigeorgis, G. Chrysos, J. Deng, and J. Shen, "The menpo facial landmark localisation challenge: A step towards the solution," in 2017 CVPRW, pp. 2116-2125.

[4] A. Bulat and G. Tzimiropoulos, "How far are we from solving the $2 \mathrm{~d} \& 3 \mathrm{~d}$ face alignment problem? (and a dataset of 230,000 3d facial landmarks)," in ICCV, 2017.

[5] J. Gu, X. Yang, S. D. Mello, and J. Kautz, "Dynamic facial analysis: From bayesian filtering to recurrent neural network," in 2017 IEEE CVPR, pp. 1531-1540.

[6] H. Zhang, Q. Li, Z. Sun, and Y. Liu, "Combining datadriven and model-driven methods for robust facial landmark detection," IEEE Inf Foren Sec, vol. 13, no. 10, pp. 24092422, 2018.

[7] H. Nada, V. A. Sindagi, H. Zhang, and V. M. Patel, "Pushing the limits of unconstrained face detection: a challenge dataset and baseline results," CoRR, vol. abs/1804.10275, 2018.

[8] Y. Zhou, D. Liu, and T. Huang, "Survey of face detection on low-quality images," in 2018 13th IEEE FG 2018, pp. 769-773.

[9] C. Qu, H. Gao, E. Monari, J. Beyerer, and J.-P. Thiran, "Towards robust cascaded regression for face alignment in the wild," in IEEE CCVPW, 2015, pp. 1-9.

[10] X. Dong, Y. Yan, W. Ouyang, and Y. Yang, "Style aggregated network for facial landmark detection," in CVPR, 2018, pp. 379-388.

[11] G. Goswami, N. Ratha, A. Agarwal, R. Singh, and M. Vatsa, "Unravelling robustness of deep learning based face recognition against adversarial attacks," in AAAI Conference on Artificial Intelligence, 2018.

[12] C. R. A. Chaitanya, A. S. Kaplanyan, C. Schied, M. Salvi, A. Lefohn, D. Nowrouzezahrai, and T. Aila, "Interactive reconstruction of monte carlo image sequences using a recurrent denoising autoencoder," ACM Trans. Graph., vol. 36, no. 4, pp. 98:1-98:12.

[13] C. Szegedy, S. Ioffe, and V. Vanhoucke, "Inception-v4, inception-resnet and the impact of residual connections on learning," CoRR, vol. abs/1602.07261, 2016.

[14] X. Zhu, Z. Lei, J. Yan, D. Yi, and S. Z. Li, "High-fidelity pose and expression normalization for face recognition in the wild," in IEEE CVPR, 2015, pp. 787-796.

[15] Y. Wu, C. Gou, and Q. Ji, "Simultaneous facial landmark detection, pose and deformation estimation under facial occlusion," arXiv preprint arXiv:1709.08130, 2017. 


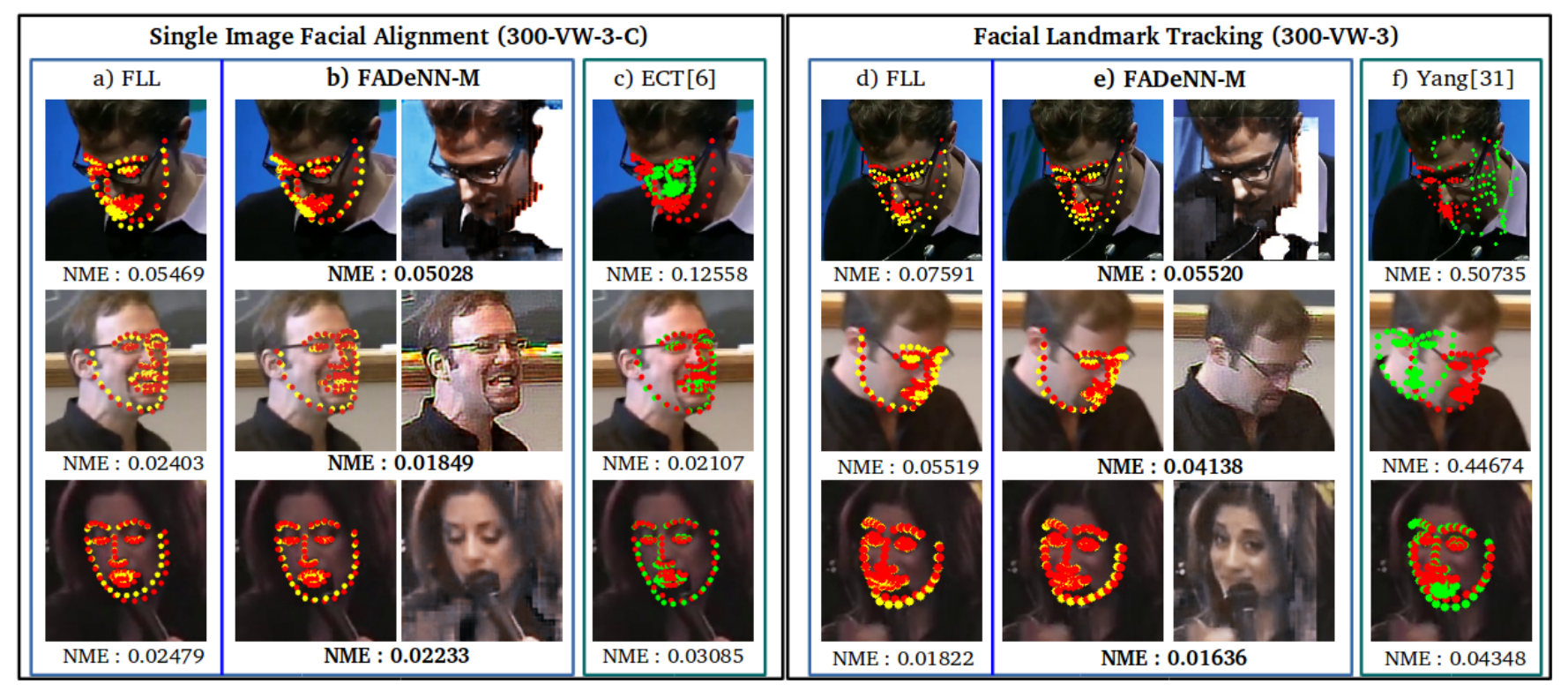

Figure 6. Visualization of single image facial landmark alignments : a) FLL, b) FADeNN-M, c)ECT[6]. Facial landmark tracking : d) FLL-T, e) FADeNN-M-T, f) Yang[31]

[16] J. Thies, M. Zollhofer, M. Stamminger, C. Theobalt, and M. Nießner, "Face2face: Real-time face capture and reenactment of rgb videos," in IEEE CVPR, 2016, pp. 2387-2395.

[17] A. L. Yuille, P. W. Hallinan, and D. S. Cohen, "Feature extraction from faces using deformable templates," Int $J$ Comput Vision, vol. 8, no. 2, pp. 99-111, 1992.

[18] T. F. Cootes, C. J. Taylor, D. H. Cooper, and J. Graham, "Active shape models-their training and application," Comput Vis Image Und, vol. 61, no. 1, pp. 38-59, 1995.

[19] V. Kazemi and J. Sullivan, "Face alignment with part-based modeling," in BMVC 2011. BMVA, 2011, pp. 27-1.

[20] M. Valstar, B. Martinez, X. Binefa, and M. Pantic, "Facial point detection using boosted regression and graph models," in IEEE CVPR 2010. IEEE, 2010, pp. 2729-2736.

[21] V. Kazemi and J. Sullivan, "One millisecond face alignment with an ensemble of regression trees," in 2014 IEEE CVPR, June 2014, pp. 1867-1874.

[22] S. Zhu, C. Li, C. C. Loy, and X. Tang, "Face alignment by coarse-to-fine shape searching," in 2015 IEEE CVPR, June 2015, pp. 4998-5006.

[23] Z. Zhang, P. Luo, C. C. Loy, and X. Tang, "Learning deep representation for face alignment with auxiliary attributes," IEEE T Pattern Anal, vol. 38, no. 5, pp. 918-930, May 2016.

[24] X. Mao, C. Shen, and Y.-B. Yang, "Image restoration using very deep convolutional encoder-decoder networks with symmetric skip connections," in NIPS. Curran Associates, Inc., 2016, pp. 2802-2810.

[25] H. Nam and B. Han, "Learning Multi-Domain Convolutional Neural Networks for Visual Tracking," 2015.
[26] Y. Zhang and Q. Yang, "A survey on multi-task learning," arXiv preprint arXiv:1707.08114, 2017.

[27] D. Aspandi, O. Pujol, F. Sukno, and X. Binefa, "Fully end-toend composite recurrent convolution network for deformable facial tracking in the wild," in 14th IEEE FG 2019, In Press.

[28] D. P. Kingma and J. Ba, "Adam: A method for stochastic optimization." CoRR, vol. abs/1412.6980, 2014.

[29] G. G. Chrysos, E. Antonakos, P. Snape, A. Asthana, and S. Zafeiriou, "A Comprehensive Performance Evaluation of Deformable Face Tracking In-the-Wild," Int J Comput Vision, pp. 1-35, 2017.

[30] K. Zhang, Z. Zhang, Z. Li, S. Member, Y. Qiao, and S. Member, "Joint Face Detection and Alignment using Multi - task Cascaded Convolutional Networks,” Spl, no. 1, pp. 1-5, 2016.

[31] J. Yang, J. Deng, K. Zhang, and Q. Liu, "Facial shape tracking via spatio-temporal cascade shape regression," in 2015 IEEE ICCVW, pp. 994-1002.

[32] M. Uricár, V. Franc, and V. Hlavác, "Facial landmark tracking by tree-based deformable part model based detector," 2015 IEEE ICCVW, pp. 963-970, 2015.

[33] S. Xiao, S. Yan, and A. A. Kassim, "Facial landmark detection via progressive initialization," in 2015 IEEE ICCVW, pp. 986 993.

[34] G. Rajamanoharan and T. F. Cootes, "Multi-view constrained local models for large head angle facial tracking," in 2015 IEEE ICCVW, pp. 971-978.

[35] Y. Wu and Q. Ji, "Shape augmented regression method for face alignment," 2015 IEEE ICCVW, pp. 979-985, 2015. 